\title{
La IL-1 y su eventual asociación con la enfermedad periodontal crónica. Una revisión de la literatura (I)
}

\author{
VALDERRAMA G* \\ VIJANDE $\mathbf{F} * *$ \\ ESCRIBANO JM*** \\ GARRIDO-PERTIERRA A**** \\ BASCONES $\mathrm{A} * * * * *$
}

Valderrama G, Vijande F, Escribano JM, Garrido-Pertierra A, Bascones A. La IL-1 y su eventual asociación con la enfermedad periodontal crónica. Una revisión de la literatura (I). Av Periodon Implantol. 2005; 17, 2: 89-95.

\begin{abstract}
RESUMEN
El propósito de esta revisión bibliográfica es describir las acciones que protagonizan las interleuquinas, con especial interés la IL-1 en el desarrollo de la enfermedad periodontal crónica, así como su presencia en el fluido gingival crevicular (FGC). La mayoría de los estudios revisados muestran una fuerte asociación entre los niveles aumentados de esta citoquina y el estado periodontal del paciente. La IL-1 puede ser un excelente marcador para detectar la severidad y progresión de la enfermedad periodontal crónica.
\end{abstract}

\section{PALABRÁS CLAVE}

Interleuquina, IL-1, enfermedad periodontal crónica.

Aceptado para publicación: Abril 2005

\section{INTRODUCCIÓN}

La enfermedad periodontal es un proceso inflamatorio de carácter crónico, desencadenado por una serie de bacterias periodonto-patógenas específicas. En este proceso inflamatorio, la interleuquina 1 (IL-1), junto a otros productos bioquímicos, son los compuestos encargados de iniciar la destrucción en los tejidos de inserción del diente. Por tal motivo, la síntesis de IL-1 está notablemente relacionada con la severidad y progresión de la enfermedad periodontal.

La enfermedad periodontal presenta una clara etiolo- gía multifactorial, donde las bacterias ejercen un factor etiológico causal e indispensable para el desarrollo de la enfermedad. Otros factores de riesgo son los biológicos y los ambientales. Dentro de los factores biológicos se encuentran las enfermedades sistémicas y la predisposición genética del individuo. Mientras que formando parte de los ambientales se observan el tabaco, el estrés y la higiene oral.

Se sabe que las reacciones inmunitarias del huésped desempeñan un papel importante en la patogenia de la enfermedad periodontal. Éstas, contribuyen en el proceso patológico y son capaces de modular los

\footnotetext{
* Odontólogo. Master en Operatoria y Estética Dental por UMS.

** Odontólogo. Master en Periodoncia e Implantes por UCM.

*** Médico. Jefe de Servicio del Instituto de Cardiología de la Comundiad de Madrid.

**** Doctor en Farmacia y en Veterinaria. Catedrático de Bioquímica y Biología Molecular del Departamento de Bioquímica y Biología Molecular IV de la Facultad de Veterinaria de la UCM.

***** Doctor Médico-Estomatólogo. Catedrático de Medicina Bucal y Periodoncia del Departamento de Medicina y Cirugía Bucofacial de la Facultad de Odontología de la UCM.
} 


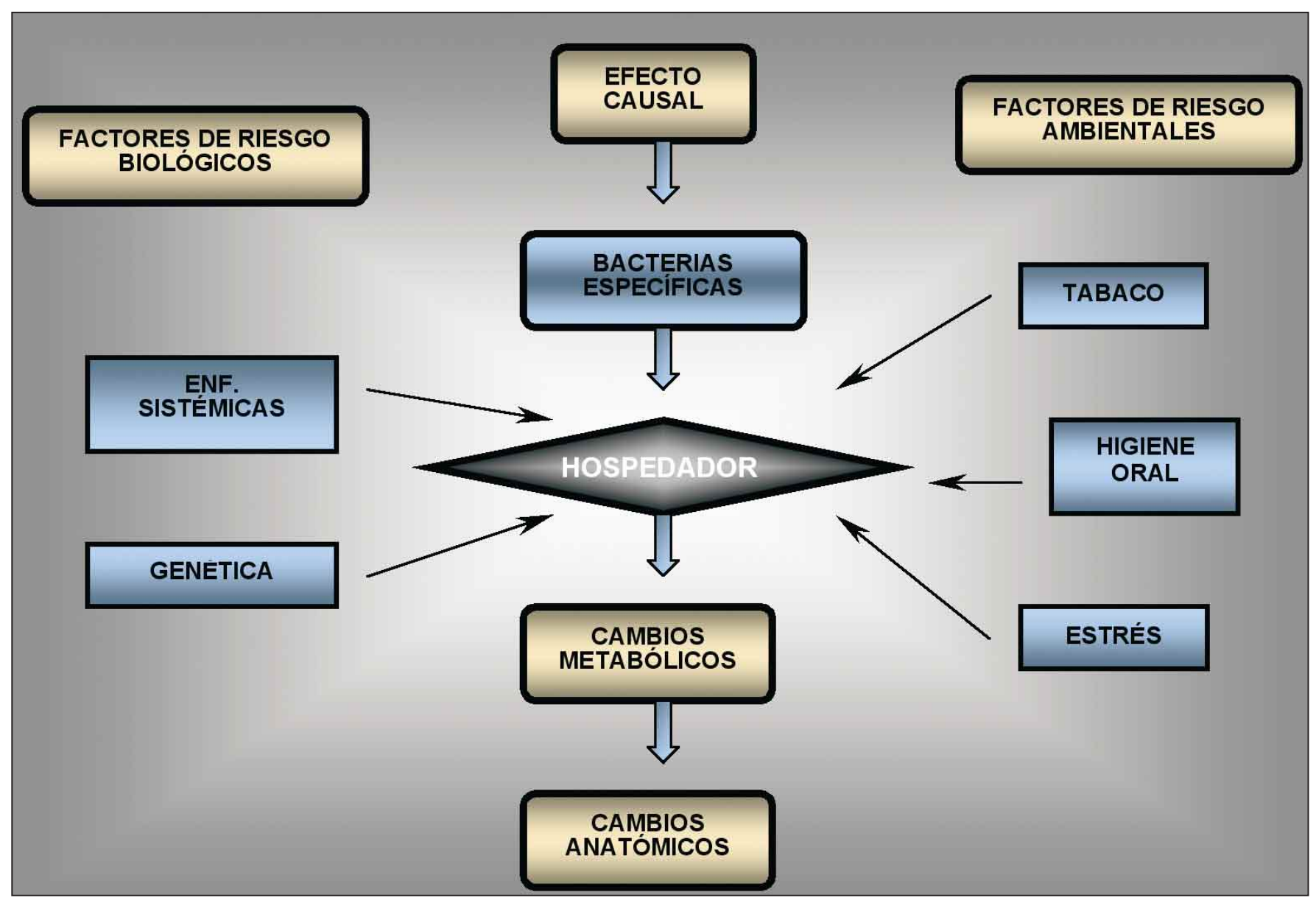

Fig. 1: Factores que intervienen en la etiología de la enfermedad periodontal.

efectos de las bacterias (1). Sin embargo, estas reacciones inmunes pueden ser beneficiosas o nocivas. Dentro del proceso destructivo de la infección, son varios los componentes del sistema inmunitario que juegan un papel destacado. Neutrófilos, linfocitos, células plasmáticas y macrófagos varían en cantidad según el estado patológico de los tejidos de inserción del diente (2).

Importancia de las citoquinas, en especial la IL-1 en la evolución de la enfermedad periodontal. Las citoquinas son proteínas solubles que median en las interacciones complejas entre los linfocitos, las células inflamatorias y otros elementos celulares del tejido conectivo. Son segregadas por células que actúan como moléculas mensajeras que transmiten señales a las otras células.

En un principio fueron llamadas linfoquinas antes de reconocer su producción por otras células. Tienen numerosas acciones como la iniciación y el mantenimiento de las respuestas inmunitaria e inflamatoria, la regulación del desarrollo y la diferenciación de las células. Las interleuquinas son miembros importantes del grupo de las citoquinas y están implicadas principalmente, en la comunicación entre los leucocitos y otras células que intervienen en los procesos inmunitarios e inflamatorios, como son las células epiteliales, las endoteliales y los fibroblastos. Estas moléculas son liberadas en pequeñas cantidades y tienen diversas acciones sobre las células que llevan el receptor específico para las citoquinas. Estas sustancias son numerosas, tienen funciones que se superponen y están ligadas entre sí formando una red activa que controla la respuesta del huésped. El control de la liberación y acción de las citoquinas es complejo y depende de inhibidores y receptores. Muchas citoquinas son capaces de actuar sobre la célula que las produce (función autocrina), de manera que autoestimulan su propia producción y la producción de otras citoquinas. Otras son las paracrinas que se fijan a células próximas, mientras que también existen las endocrinas capaces de unirse a células distantes.

Las citoquinas pueden ser pleiotróficas, provocando diferentes actividades biológicas a partir de distintas 
células. Por otra parte, diferentes citoquinas pueden mostrar reacciones similares $(3,4)$. La acumulación de células plasmáticas y linfocitos en los tejidos periodontales sugiere que las citoquinas intervienen en los cambios patológicos del periodonto. Inicialmente, las citoquinas fueron denominadas según su actividad biológica. Estas incluyeron al factor activador de macrófagos (MAF, macrophage activation factor), el de inhibición de migración de macrófagos (MIF, migration inhibition factor), el factor quimiotáctico derivado de leucocitos (CTX, leukocyt derived chemotactic factor), la linfotaxina (LT) y el factor activador de osteoclastos (OAF, Osteoclast-activating factor) (5).

Citoquinas proinflamatorias. Las citoquinas proinflamatorias son capaces de estimular a los macrófagos y a otras células para producir cantidades importantes de prostaglandinas, en particular PGE2, que son potentes vasodilatadores e inductores a su vez, de la producción de citoquinas por diversas células. Las prostaglandinas actúan sobre los fibroblastos y osteoclastos, junto con las citoquinas, para inducir la producción de metaloproteinasas de la matriz, lo cual es relevante para el recambio tisular y para el proceso destructivo periodontal.

La interleuquina-1 (IL-1), la IL-6 y el factor de necrosis tumoral (TNF) estimulan la reabsorción ósea in vitro e in vivo. Las actividades de estas tres citoquinas han sido estudiadas en muestras de fluído gingival crevicular (FGC) en zonas con inflamación clínica en seres humanos. Otros estudios también intentaron determinar los niveles de citoquinas en tejidos sanos y enfermos. Investigaciones in vitro en cuanto al mecanismo de acción de la IL-1 sobre los fibroblastos, sugieren que la IL-1 puede actuar sobre los fibroblastos para promover la reparación o la destrucción de la matriz celular. También demostraron que el material del implante dental puede estimular a las células mononucleares periféricas para producir IL-1 y TNF, lo que, posteriormente en teoría, da por resultado una reabsorción ósea y la consiguiente pérdida del implante. Las consecuencias clínicas sin embargo, no son tan evidentes (6).

Citoquinas quimiotácticas. Se han identificado una serie de más de 20 moléculas entre las cuales la más famosa y mejor caracterizada es la inteleuquina-8 (IL8), que tiene poderosas funciones quimiotácticas para los leucocitos, particularmente para los neutrófilos, pero también para los linfocitos y macrófagos. Estas moléculas actúan reclutando células de defensa hacia áreas donde se las necesita y son importantes en las respuestas con mediación celular. Se utiliza el término quimioquina para describir estas moléculas y es una forma abreviada de "citoquina quimiotáctica" (6).

Citoquinas señaladoras de linfocitos. Los linfocitos
T colaboradores (TH1 y TH2) son linfocitos que regulan en los tejidos las respuestas inmunitarias humoral y de mediación celular por la vía de las citoquinas. La respuesta de inmunidad humoral es promovida por TH2, que produce citoquinas características, a saber, IL-4, IL-5, IL-10 e IL-13. Los linfocitos TH1 liberan IL-2 e interferón (IFN)-IFN $\gamma$, que refuerzan las respuestas de mediación celular. Estas citoquinas constituyen un mecanismo preciso para el control de la respuesta inmunitaria, de modo que sea suficiente para enfrentarse al patógeno. Las citoquinas pueden influir sobre la respuesta inmunitaria, determinando la clase de inmunoglobulina producida, lo que puede tener un efecto profundo sobre la función de los anticuerpos. Por ejemplo, las moléculas IgM son más eficaces para la bacteriolisis mientras que las moléculas IgG lo son para la opsonización.

Existen cuatro tipos diferentes de anticuerpos IgG $\left(\operatorname{IgG}_{1-4}\right)$ basados en diferencias en la porción $\mathrm{FC}_{\mathrm{C}} \mathrm{de}$ estas moléculas. La subclase anticuerpo influye sobre la función anticuerpo, con la $\operatorname{IgG}_{2}$ como antígeno de unión menos fuerte que la IgG. Varios investigadores hallaron la cantidad de $\operatorname{IgG}_{2}$ superior al lado de la $\mathrm{IgG}_{1}$ en pacientes con periodontitis grave y proponen que los niveles de la subclase IgG son factores importantes en la susceptibilidad a la periodontitis (7). Yamazaki y col. $(8,9)$, publicaron que los linfocitos productores de TH2 predominan en las lesiones de gingivitis y periodontitis. Las células TH2 refuerzan la respuesta inmunitaria humoral antes que las respuestas mediadas por células. Esto avala el concepto de que estas lesiones son dominadas por plasmocitos y además, que la respuesta inmunitaria humoral es más notable que la respuesta de mediación celular en la periodontitis crónica (6).

Más recientemente, la mayor parte de las citoquinas de nuevo se denominaron interleuquinas, en referencia a su función en la comunicación interleucocitaria. Hoy en día, más de 10 interleuquinas se han identificado. Otras incluso reciben su nombre sobre la base de su actividad biológica (3).

IL-1 ( $\alpha$ y $\beta)$. Es una citoquina pleiotrófica con diversas actividades. Incluye a $\operatorname{OAF}(4,10,11)$ debido a su estimulación de osteoclástos y al factor activador de linfocitos (LAF, lymphocyteactivating factor) en vista de su capacidad para estimular la proliferación de células $\mathrm{T}$ tratadas con fitohemaglutinación. Se sabe también que interviene en la activación de células TH, el fomento de la maduración de células $\mathrm{B}$, la quimiotaxis de neutrófilos y macrófagos, la mejoría de la actividad de células NK, así como en otras reacciones.

La IL-1 secretada por monocitos, macrófagos, células B, fibroblastos, neutrófilos, células epiteliales y muchos otros tipos de células estimuladas. Esta esti- 
mulación causa fagocitosis, componentes del complemento (C3a y C5a), otras diferentes sustancias. La IL-1 aparece en los tejidos gingivales y en el líquido del surco, y decrece después del tratamiento periodontal $(12,13,14)$. También aumenta el procolágeno de fibroblastos, la protaglandina E2 (PGE2) y la actividad de la reabsorción ósea (15). La interleuquina l (IL-1), citoquina producida por macrófagos, células B y células del epitelio escamoso, es un importante mediador inmunitario. Su liberación es estimulada a partir de estas células por lipooligosacáridos (LOS) de periodontopatógenos como: P.gingivalis y C.rectus. La IL-1 motiva la proliferación de los timocitos, las células T, las células B y los fibroblastos, así como otras células. Además, mejora la producción de linfocinas, entre ellas el factor de crecimiento de células T (IL-2) y el factor activador de osteoclastos (16). Así mismo la IL1 mejora la producción de anticuerpos por las células $\mathrm{B}$, y de colagenasa y prostaglandinas por los fibroblastos. La IL-1 aparece en mayores cantidades en el líquido gingival de lugares inflamados; lo que sugiere que podría intervenir en la enfermedad periodontal influyendo en las reacciones inmunitaria e inflamatoria del huésped ante los mitógenos y antígenos bacterianos $(17,18)$.

Relación entre la [IL-1 $\beta$ ] en FGC y el estado periodontal según diferentes estudios. Preiss y Meyle (19), al igual que Rawlison y col. $(20,21)$ fueron capaces de hallar valores de IL-1 $\beta$ en FGC en todas las localizaciones estudiadas, independientemente de pertenecer a un grupo sano o a uno con enfermedad periodontal. De igual manera, tanto en las localizaciones sanas como en las enfermas se detectó esta citoquina. Por el contrario otros autores $(22,23,24)$ no fueron capaces de detectar valores de IL- $1 \beta$ en pacientes sanos. Estas discrepancias pueden deberse a los diferentes métodos de recolección de muestras y/o al empleo de diferentes kits de ELISA.

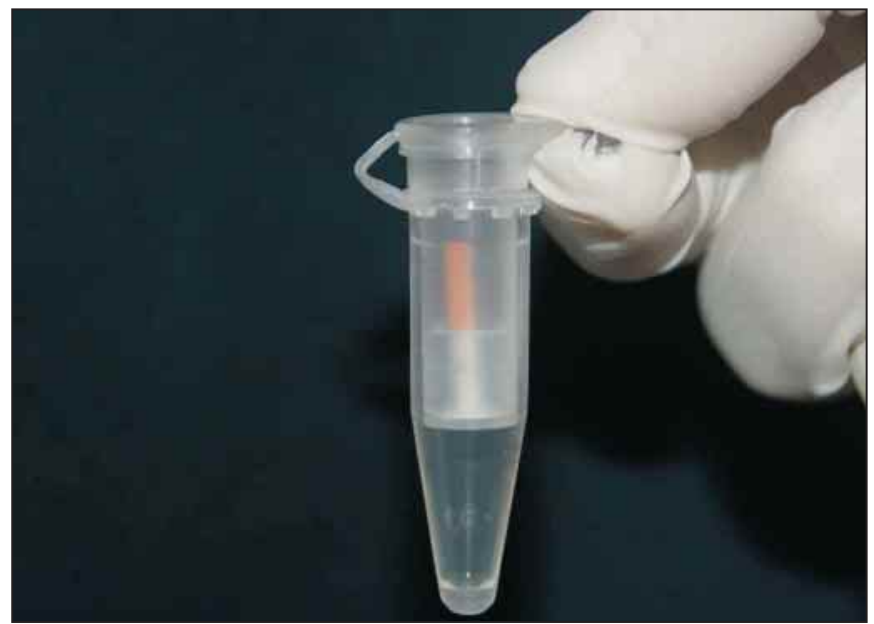

Fig. 2: Muestra del fluido gingival crevicular.
Actualmente no existe ninguna "gold standard" para identificar localizaciones con actividad de la enfermedad periodontal. Un criterio bastante fiable es el denominado método de la tolerancia descrito por Listgarten (25), quien emplea la pérdida de inserción como parámetro valorable. Considera activa una bolsa periodontal, cuando en un intervalo de dos meses se produce un aumento de $\geq 2.5 \mathrm{~mm}$ en la pérdida inserción. Pero la única conclusión que se puede obtener es que en algún momento dentro de ese intervalo de tiempo, la bolsa periodontal estuvo activa. Otro criterio empleado en estudios transversales para determinar la actividad de la bolsa periodontal es el descrito por Lang y col. (26) que se basa en determinar la actividad de la bolsa según la presencia o ausencia del sangrado al sondaje.

Varios estudios $(27,28,29,30,31)$ donde se registraron las concentraciones de IL-1 $\beta$ en FGC tanto en pacientes con enfermedad periodontal como en pacientes sanos, fueron capaces de encontraron diferencias estadísticamente significativas entre ambos grupos. Observándose las [IL-1 $\beta$ ] más elevadas en el grupo con enfermedad periodontal activa. Estas concentraciones eran aproximadamente del doble en el grupo con enfermedad periodontal. Los autores ponen de manifiesto que la IL-1 $\beta$ está fuertemente relacionada con la condición inflamatoria del periodonto. Stashenko y col. (32) en un estudio longitudinal, observaron que aquellos lugares que presentaban una pérdida de inserción $\geq 2.5 \mathrm{~mm}$ en un intervalo de dos meses, poseían los niveles más elevados de IL$1 \beta$. Estos niveles eran 2.5 veces mayores que en las localizaciones enfermas sin una pérdida de inserción tan marcada, y de 4.6 veces mayores que en las localizaciones sanas. En otro estudio similar sobre 15 pacientes con enfermedad periodontal de caracter moderada-severa, Masada y col. (23) además de detectar valores de IL- $1 \alpha$ y de IL- $1 \beta$ en FGC, obser-

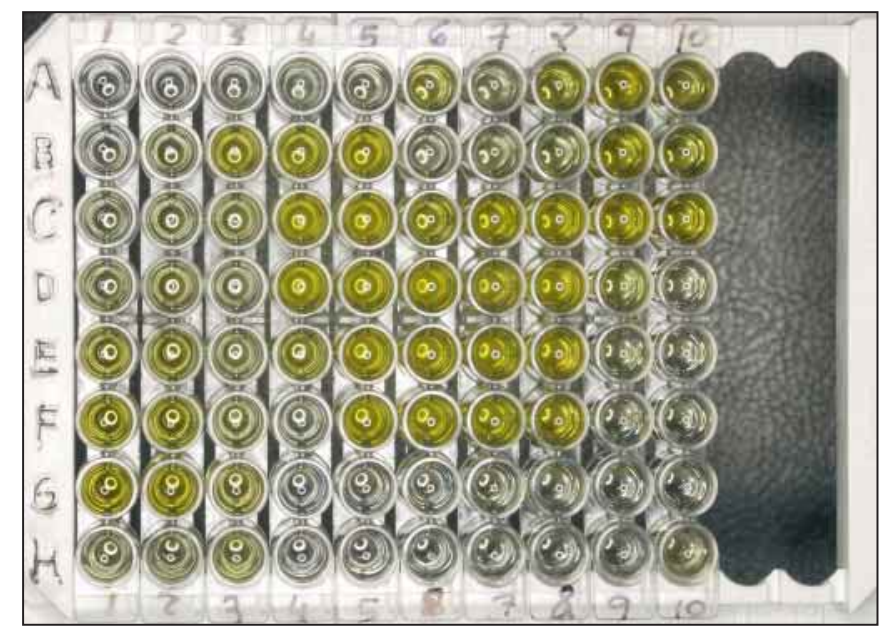

Fig. 3: Resultados con el kit de ELISA. 
varon como esos valores disminuyeron después de recibir el tratamiento periodontal básico. Salvi y col. (33) también detectaron niveles de IL-1 $\beta$ en FGC aumentados en pacientes con periodontitis moderada-severa cuando los compararon frente a pacientes con gingivitis y periodontitis leve. Tanto Faizuddin y col. (34) como Preiss y Meyle (19) observaron que los valores de IL-1 $\beta$ en FGC de pacientes con gingivitis sin pérdida y con pérdida de inserción periodontal eran mayores que el los de los pacientes periodontalmente sanos.

Rawlison y col. (20) tomaron una serie de precauciones para evitar que las muestras de IL-1 $\beta$ en FGC se contaminasen con saliva o con sangre, ya que según Dinarello 1991 (35) y Tizard 1995 (21) estos dos fluídos contienen cantidades de IL-1 $\beta$ y podrían alterar los datos obtenidos. Tras localizar las bolsas activas sangrantes, antes de tomar la muestra se esperó el tiempo necesario hasta que la lesión dejase de sangrar y además, para evitar la posible contaminación con saliva, se procedió a aislar la zona con rollos de algodón, se empleó el eyector de saliva y se decidió elegir dientes dentales del maxilar en vez de la mandíbula. Dichos autores en su estudio dejaban durante tres minutos la tira de papel en el interior del surco, siguiendo las indicaciones a las que llegaron Reinhardt y col. (36), estos autores propusieron que el tiempo de espera de 30 segundos era insuficiente para tomar una cantidad de muestra adecuada. Observaron que las [IL-1 $\beta$ ] en FGC más elevadas las obtuvieron las localizaciones profundas y sangrantes, los valores intermedios los poseían las localizaciones profundas y no sangrantes y por último, las localizaciones sanas mostraron los niveles más bajos. Por el contrario, Rawlison y col. (37) tres años más tarde en un estudio similar donde incluyeron un grupo fumador y otro no fumador, observaron que las [IL-1 $\beta$ ] en FGC más bajas se encontraban en las localizaciones con enfermedad periodontal. Esto sucedía en ambos grupos. Por el contrario, los valores más elevados se encontraban en las localizaciones sanas. Dichos autores sugieren que estas diferencias pueden deberse a la recalibración del periotrón. Ésta se llevó a cabo con una mezcla de sueros, en lugar de con suero salino como en el primer estudio. Añaden que actualmente el fluído más indicado para la calibración del periotrón es la mezcla de sueros.

Engebreston y col. (38) en un estudio longitudinal de 24 semanas, registraron los niveles de IL-1 $\beta$ en FGC en baseline y los compararon después de realizar el tratamiento periodontal básico, que consistió en raspado y alisado radicular. Los niveles más elevados se registraron en pacientes con enfermedad periodontal severa y los más bajos en pacientes con enfermedad periodontal leve. A las dos semanas del tratamiento, las [IL-1 $\beta$ ] en FGC se redujeron en todos los pacien- tes. A las 24 semanas, esa [IL-1 $\beta$ ] se mantuvo disminuida en los pacientes con una enfermedad periodontal más leve, mientras que en los pacientes con enfermedad periodontal más severa, estos niveles volvieron a recuperar los valores previos al tratamiento. Cuando la profundidad de sondaje se contempló como una variable en el registro de IL-1 $\beta$, se observó que los niveles más elevados se encontraban en las bolsas menos profundas. Giannopoulou y col. (39) también observaron que los niveles más elevados de IL- $1 \beta$ en FGC los poseían los grupos con enfermedad periodontal al compararse con los grupos de gingivitis y de control. Además, las localizaciones activas en los grupos con periodontitis, presentaban niveles más elevados de IL-1 $\beta$ en FGC que los de las localizaciones inactivas. Tanto la IL-1 $\beta$, como la IL-6 y la IL-8 están asociadas con la destrucción periodontal. Por el contrario, la IL-4 muestra un efecto protector respecto al estado periodontal, encontrándose los valores más altos en las localizaciones sanas. No se encontró ningún tipo de asociación entre el hecho de ser un paciente fumador y los niveles de IL-1 $\beta$ en FGC, pero se encontró con respecto al estrés, viéndose aumentados los niveles de IL-1 $\beta$, de IL-6 y de IL-8 en pacientes con estrés.

Masada y col. (23), no encontraron ningún tipo de correlación entre los niveles de IL-1 $\beta$ en FGC y la profundidad de la bolsa periodontal. Manifiestan que esta falta de correlación era previsible, ya que la profundidad de la bolsa periodontal refleja la evolución acumulada en la historia de la enfermedad, y no tiene que mostrar la actividad de la periodontitis. Wilton y col. (24) tampoco encontraron ningún tipo de correlación entre la concentración de IL-1 $\beta$ en FGC y los parámetros clínicos que estudiaron: profundidad de sondaje, pérdida de inserción e índices de sangrado y placa. Por el contrario Hou y col. (40), observaron que la cantidad total, pero no la concentración de IL$1 \beta$ en FGC correlaciona tanto con el índice gingival como con la profundidad de sondaje. Así mismo Engebreston y col. (38) también observaron una asociación estadísticamente significativa entre la [IL-1 $\beta$ ] en FGC y la profundidad al sondaje, así como también con la pérdida de inserción. Así mismo Cavanaugh y col. (41) hallaron una correlación entre los niveles de IL-1 $\beta$ en FGC y la máxima pérdida de hueso en altura.

En conclusión, después de analizar los resultados obtenidos por diversos autores en numerosos estudios, existe cierta discrepancia en lo que respecta a la asociación entre la prevalencia de la enfermedad periodontal y el aumento en los niveles de IL-1 $\beta$ en FGC. Aunque la tendencia en la mayoría de los estudios, es confirmar esta asociación. Se demuestra así la importancia que manifiesta la IL-l en la severidad y progresión de la enfermedad periodontal. 


\section{SUMMMARY}

The aim of this bibliographic review is describing the actions played by the interleukins, especially IL- 1 in development of chronic periodontal disease and it's presence in gingival crevicular fluid (GCF). Most of the reviewed articles show a strong relationship between high levels of these cytokines and the periodontal status of the patient. Thus, IL-1 becomes in en excellent marker for detecting the severity and progression of chronic periodontal disease.

\section{KEY WORDS}

Interleukin, IL-1, chronic periodontal disease.

\section{BIBLIOGRAFÍA}

1. Nisengard RJ. The role of immunology in periodontal disease. J Periodontol 1977; 48: 505-9.

2. Taubman MA, Ebersole JL, Smith DJ. Association between systemic and local antibody and periodontal disease. In Genco RJ, Mergenhagen SE, eds, Host Parasite Interactions in Periodontal Disease. Washington, DC, American Society for Microbiology 1982: 283-7.

3. Kuby J. Immunology. New York, WH Freeman 1992.

4. Morley H. Prostaglandins and lymphokines in arthritis. Prostaglandins 1974;8: 315-20.

5. Mundy GR. Inflammatory mediators and the destruction of bone. J Periodont Res 1991; 26: 213-7.

6. Lindhe J. Periodontología clínica e implantología odontológica. Tercera edición Ed: panamericana 2000 capítulos: 5 y 13.

7. Wilson ME, Bronson PM, Hamilton RG. Immunoglobulin G2 antibodies promote neutrophil killing of Actinobacillus actinomycetemcomitans. Infection and Immnity 1995; 63: 1070-5.

8. Yamazaki K, Nakajima T, Gemmell E, Polak B, Seymour GJ, Hara K. IL-4 and IL-6 producing cells in human periodontal disease tissue. Journal of Oral Pathology and Medicine 1994; 23: 347-53.

9. Yamazaki K, Nakajima T, Hara K. Immunohistological analysis of $\mathrm{T}$ cells functional subsets in chronic inflammatory periodontal disease. Clinical Experimental Immunology 1995; 99: 384-91

10. Gemsa D, Steggemann L, Menzel J. Release of cylic AMP from macrophages by stimulation with prostaglandins. J Immunol 1975;144: 1422-1428
11. Ranney RR. Immunologic mechanisms of pathogenesis in periodontal diseases: An assessment. J Periodont Res $1991 ; 26: 243-8$

12. Charon JA, Luger TA, Mergenhagen S, Oppenhein JJ. Increased thymocyte activating factor in human gingival fluid during gingival inflammation. Infect Immun 1982; 38: 1190-5.

13. Malberg K, Mölle A, Streuer D, Gängler P. Determination of lymphocyte populations and subpopulations extracted from chronically imflamed periodontal tissues. J Clin Periodontol 1992; 19: 155-9.

14. McFarlane CG, Meikle MC. Interleukin-2, interleukin-2 receptor and interleukin-4 levels are evaluated in the sera of patients with periodontal disease. J Periodont Res 1990; 25: 156-61.

15. Genco RJ. Host responses in periodontal tissues: Current concepts. J Periodontol 1984; 63: 441-6.

16. Seymour GF, Cole KL, Powell RN. Interleukin-2 production and bone resorption activity in vitro by unstimulated lymphocytes extracted from chronically inflamed human periodontal tissues. Arch Oral Biol 1985; 30: 481-7.

17. Page RC. The role of inflammatory madiators in the pathogenesis of periodontal disease. J Periodont Res 1991; 26: $230-5$.

18. Carranza FA, Newman MG. Periodontología clínica Octava edición Ed: McGraw-Hill Interamericana 1997 capítulos: 8 y 9 .

19. Preiss DS, Meyle J. Interleukin-1 beta concentration of gingival crevicular fluid. J Periodontol 1994; 65: 423-8.

20. Rawlinson A, Dalati MHN, Rahman S, Fairclough AL. Interleukin-1 and IL-1 receptor antagonist in gingival crevicular fluid. J Clin Periodontol 2000; 27: 738-43.

21. Tizard IA. Immunology: an introduction, 4th edn. Florida: Saunders Colege Publishing.

22. Altman LC, Page RC, Vandesteen GE, Dixon LI, Bradford C. Abnormalities of leukocyte chemotaxis in patients with various forms of periodontitis. J Periodont Res 1985; 20: 553-63.

23. Masada MP, Persson R, Kenney JS, Lee SW, Page RC Allison AC. Measurement of interleukin-1 alpha and 1 beta in gingival crevicular fluid: implications for the pathogenesis of periodontal disease. J Periodont Res 1990; 25: $156-63$

24.Wilton JMA, Bampton JLM, Griffiyhs GS. Interleukin-1 beta (IL-1 $\beta$ ) levels in gingival crevicular fluid: Implcations for the pathogenesis of periodontal disease. J Clin Periodontol 1992; 19: 53-7.

25. Listgarten MA. A prospective on periodontal diagnosis. J Clin Periodontol 1986; 13: 175-81.

26. Lang NP, Joss A, Orsanic T, Gusberti FA, Siegrist BE. Bleeding on probing. A predictor for the progression of periodontal disease. J Clin Periodontol 1986; 13: 590-6. 
27. Lee HJ, Kang IK, Chung CP, Choi SM. The subgingival microflora and gingival crevicular fluid cytokines in refractary periodontitis. J Clin Periodontol 1995; 22: 88590.

28. Ishihara Y, Nishihara T, Koroyanagi T, Shirozu N, Yamagishi E, Ohguchi M, Koide M, Ueda N, Amano K, Noguchi T. Gingival crevicular interleukin-l and interleukin-l receptor antagonist levels in periodontally healthy and diseased sites. J Periodont Res 1997; 32: 524-9.

29. Pongsawat Suwatanapongched, Penpan Laohapand, Rudee Surarit, Yasukazu Ohmoto, Kiat Ruxrungtham. Interleukin-1 $\beta$ level in gingival crevicular fluid of patients with active periodontitis. Asian Pacific J of allergy and immunology 2000;18: 201-7.

30. Gamonal J, Jorge O, Silva A. Interleuquina-1 $\beta$ e interleuquina-8 en pacientes adultos con periodontitis destructiva: efectos del tratamiento periodontal. Av. Periodon Implantol O 1999; 11: 183-93.

31. Figueredo CMS, Ribeiro MSM. Fischer RG, Gustafson A. Increased intreleukin-1 beta concentration in gingival crevicular flui as a character of periodontitis. J Periodontol 1999; 70: 1457-63.

32. Stashenco P, Fujiyoshi P, Obernesser MS, Prostak L, Haffajee $A D$, Socransky SS. Levels of interleukin $1 \beta$ in tissue from sites of active periodontal disease. J Clin Periodontol 1991; 18: 548-54.

33. Salvi GE, Yalda B, Collins JG. Inflammatory mediator respon-se as a potencial risk marker for periodontal diseases in insulindependent diadetes mellitus patients. J Periodontol 1997; 68: 127-35.

34. Faizuddin M, Bhararathi SH, Rohini NV. Estimation of interleukin-1 $\beta$ levels in the gingival crevicular fluid in health and inflammatory periodontal disease. J Periodont Res 2003; 38: 111-4.

35. Dinarello CA. Interleukin-1 and interleukin-1 antagonism. Blood 1991; 77: 1627-52.
36. Reinhardt RA, Masada MP, Kaldahl WB, Du Bois LM, Kornman KS, Choi JI, Kalk-warf KL, Allison A. Gingivl fluid IL-1 and IL-6 levels in refractary peiodontitis. J Clin Periodontol 1994; 20: 225-31.

37. Rawlinson A, Grummitt JW, Walsh TF, Douglas CWI. Interleukin 1 and receptor antagonist levels in gingival crevicular fluid in heavy smokers versus non-smokers J Clin Periodontol 2003; 30: 42-8.

38. Engebreston SP, Grbic JY, Singer R, Lamster IB. GCF IL-1 $\beta$ profiles in periodontal disease. J Clin Periodontol 2002; 29: 48-53.

39. Giannopoulou C, Kamma JJ, Mombelli A. Effect of inflammation, smoking and stress on gingival crevicular fluid cytokine levels. J Clin Periodontol 2003; 30: 145-53.

40. Hou LT, Liu CM, Rossomando EF. Crevicular interleukin$1 \beta$ in moderate and severe periodontitis patients and the effect of phase I periodontal treatment. J Clin Periodontol 1995; 22: 162-7.

41. Cavanaugh PF, Meredith MP, Buchanan W, Doyle MJ, Reddy MS, Jeffcoat MK. Coordinate production of PGE2 and IL-1 $\beta$ in the gingival crevicular fluid in adults with periodontitis: its relationship to alveolar bone loss and disruption by daily treatment with ketolacromethamine oral rinse. J Periodont Res 1998; 33: 75-82.

\section{CORRESPONDENCIA}

Guillermo Valderrama Mateos

c/ Porvenir $n^{\circ} 4$ bajo A colmenarejo Madrid cp:28270 telf. 918589061 / 649434868 